\title{
Limiting two-Higgs-doublet models
}

\section{Alessandro Broggio, ${ }^{a}$ Eung Jin Chun, ${ }^{b}$ Massimo Passera, ${ }^{c}$ Ketan M. Patel ${ }^{c}$ and Sudhir K. Vempati ${ }^{d}$}

\author{
${ }^{a}$ Paul Scherrer Institut, \\ CH-5232 Villigen, Switzerland \\ ${ }^{b}$ Korea Institute for Advanced Study, \\ Seoul 130-722, Korea \\ ${ }^{c}$ INFN - Sezione di Padova, \\ I-35131 Padova, Italy \\ ${ }^{d}$ Centre for High Energy Physics, Indian Institute of Science, \\ Bangalore 560012, India \\ E-mail: alessandro.broggio@psi.ch, ejchun@kias.re.kr, \\ passera@pd.infn.it, ketan.patel@pd.infn.it, vempati@cts.iisc.ernet.in
}

Abstract: We update the constraints on two-Higgs-doublet models (2HDMs) focusing on the parameter space relevant to explain the present muon $g-2$ anomaly, $\Delta a_{\mu}$, in four different types of models, type I, II, "lepton specific" (or X) and "flipped" (or Y). We show that the strong constraints provided by the electroweak precision data on the mass of the pseudoscalar Higgs, whose contribution may account for $\Delta a_{\mu}$, are evaded in regions where the charged scalar is degenerate with the heavy neutral one and the mixing angles $\alpha$ and $\beta$ satisfy the Standard Model limit $\beta-\alpha \approx \pi / 2$. We combine theoretical constraints from vacuum stability and perturbativity with direct and indirect bounds arising from collider and $B$ physics. Possible future constraints from the electron $g-2$ are also considered. If the $126 \mathrm{GeV}$ resonance discovered at the LHC is interpreted as the light CP-even Higgs boson of the 2HDM, we find that only models of type $\mathrm{X}$ can satisfy all the considered theoretical and experimental constraints.

Keywords: Higgs Physics, Beyond Standard Model

ARXiv EPRINT: 1409.3199 


\section{Contents}

1 Introduction 1

2 Electroweak constraints 3

3 Theoretical constraints on the splitting $M_{A^{-}} M_{H^{+}} \quad 5$

4 Constraints from the muon $g-2 \quad 7$

5 Constraints from the electron $g-2 \quad 10$

6 Conclusions 11

\section{Introduction}

The ATLAS and CMS Collaborations at the LHC [1, 2] found a neutral boson with a mass of about $126 \mathrm{GeV}$ which confirms the Brout-Englert-Higgs mechanism. It is now of imminent interest to check whether this new boson is the unique one following exactly the Standard Model (SM) prediction, or if there are other bosons participating in the electroweak (EW) symmetry breaking. One of the simplest way to extend the SM is to consider two Higgs doublets participating in the EW symmetry breaking instead of the standard single one. There are in fact several theoretical and experimental reasons to go beyond the SM and look forward to non-standard signals at the next run of the LHC and at future collider experiments. For reviews on two-Higgs-doublet models, see [3, 4].

A major constraint to construct models with two Higgs doublets (2HDMs) arises from flavour changing neutral currents, which are typically ubiquitous in these models. Requiring Natural Flavour Conservation (NFC) restricts the models to four different classes which differ by the manner in which the Higgs doublets couple to fermions [4-6]. They are organized via discrete symmetries like $Z_{2}$ under which different matter sectors, such as right-handed leptons or left-handed quarks, have different charge assignments. These models are labeled as type I, II, "lepton-specific" (or X) and "flipped" (or Y). Normalizing the Yukawa couplings of the neutral bosons in such a way that the explicit Yukawa interaction terms in the Lagrangian are given by $\left(y_{f}^{\phi}\right) \frac{m_{f}}{v} \bar{f} f \phi$ for the CP-even scalars $\phi=h, H$ (lighter and heavier, respectively) and $i\left(y_{f}^{A}\right) \frac{m_{f}}{v} \hat{f} \gamma_{5} f A$ for the pseudoscalar $A$ in the mass eigenstate basis, the $y_{f}^{h, H, A}$ factors are summarized in table I for each of these four types of $2 \mathrm{HDMs}$ as functions of $\tan \beta \equiv v_{2} / v_{1}$, the ratio of the two Higgs vacuum expectation values, and the diagonalization angle $\alpha$ of the two CP-even Higgs bosons $\left(v=\sqrt{v_{1}^{2}+v_{2}^{2}}=246 \mathrm{GeV}\right)$. However it should be noted that in addition to these models, NFC can also occur in models with alignment, as in ref. [7]. In this class of models, more general sets of relations are 


\begin{tabular}{|l|ccccccccc|}
\hline & $y_{u}^{A}$ & $y_{d}^{A}$ & $y_{l}^{A}$ & $y_{u}^{H}$ & $y_{d}^{H}$ & $y_{l}^{H}$ & $y_{u}^{h}$ & $y_{d}^{h}$ & $y_{l}^{h}$ \\
\hline Type I & $\cot \beta$ & $-\cot \beta$ & $-\cot \beta$ & $\frac{\sin \alpha}{\sin \beta}$ & $\frac{\sin \alpha}{\sin \beta}$ & $\frac{\sin \alpha}{\sin \beta}$ & $\frac{\cos \alpha}{\sin \beta}$ & $\frac{\cos \alpha}{\sin \beta}$ & $\frac{\cos \alpha}{\sin \beta}$ \\
Type II & $\cot \beta$ & $\tan \beta$ & $\tan \beta$ & $\frac{\sin \alpha}{\sin \beta}$ & $\frac{\cos \alpha}{\cos \beta}$ & $\frac{\cos \alpha}{\cos \beta}$ & $\frac{\cos \alpha}{\sin \beta}$ & $-\frac{\sin \alpha}{\cos \beta}$ & $-\frac{\sin \alpha}{\cos \beta}$ \\
Type X & $\cot \beta$ & $-\cot \beta$ & $\tan \beta$ & $\frac{\sin \alpha}{\sin \beta}$ & $\frac{\sin \alpha}{\sin \beta}$ & $\frac{\cos \alpha}{\cos \beta}$ & $\frac{\cos \alpha}{\sin \beta}$ & $\frac{\cos \alpha}{\sin \beta}$ & $-\frac{\sin \alpha}{\cos \beta}$ \\
Type Y & $\cot \beta$ & $\tan \beta$ & $-\cot \beta$ & $\frac{\sin \alpha}{\sin \beta}$ & $\frac{\cos \alpha}{\cos \beta}$ & $\frac{\sin \alpha}{\sin \beta}$ & $\frac{\cos \alpha}{\sin \beta}$ & $-\frac{\sin \alpha}{\cos \beta}$ & $\frac{\cos \alpha}{\sin \beta}$ \\
\hline
\end{tabular}

Table 1. The normalized Yukawa couplings of the neutral bosons to up- and down-type quarks and charged leptons.

imposed on the field content using discrete symmetries similar to $Z_{2}$, which still conserve flavour but allow for $\mathrm{CP}$ violation. A class of $2 \mathrm{HDMs}$ also exists where one of the Higgs doublets does not participate in the dynamics and remains inert [8, 9]. Finally, in the so-called type III models both up and down fermions couple to both Higgs doublets. A detailed analysis of flavour and CP violation in type III models can be found in [10] and references therein.

One of the possible experimental indications for new physics is the measurement of the muon $g-2\left(a_{\mu}\right)$, which at present shows a $3-3.5 \sigma$ discrepancy $\Delta a_{\mu}$ from the SM prediction. Although not large enough to claim new physics, $\Delta a_{\mu}$ can be used as a guideline to single out favourable extensions of the SM. In this paper we will study if such a deviation can be accounted for in 2HDMs of types I, II, X, and Y. A contribution to $a_{\mu}$ able to bridge the $\Delta a_{\mu}$ discrepancy can arise in 2HDMs from a light pseudoscalar through Barr-Zee type two-loop diagrams [11-15]. However, a light pseudoscalar may be in conflict with a heavy charged scalar whose mass is strongly constrained by direct and indirect searches. In fact, the general 2HDM lower bound on the mass of the charged scalar $H^{ \pm}$from direct searches at LEP2 is $M_{H^{ \pm}} \gtrsim 79 \mathrm{GeV}$ [16], and even stronger indirect bounds can be set from $B$-physics in type II and Y models.

In $2 \mathrm{HDMs}$, the observed $126 \mathrm{GeV}$ resonance can be identified with any of the two CP-even Higgs bosons. ${ }^{1}$ In the present paper we identified this resonance with the lightest CP-even scalar $h$. This interpretation is possible in all four 2HDMs types considered here. In particular, we chose the limit $\beta-\alpha=\pi / 2$ in which the couplings of the light CP-even neutral Higgs $h$ with the gauge bosons and fermions attain the SM values. In fact, the measured signal strengths and production cross section of such a particle are in very good agreement with the corresponding SM predictions [18-34].

In addition to the bounds set by the muon $g-2,2 \mathrm{HDMs}$ are constrained by direct searches at colliders for the Higgs bosons $h, H, A$ and $H^{ \pm}, B$-physics observables, EW precision measurements and theoretical considerations of vacuum stability and perturbativity. The question then arises: which of these models are preferred by the present set of direct and indirect constraints? In this work we addressed this question concentrating on the

\footnotetext{
${ }^{1}$ In this paper, we work in the CP-conserving case i.e, we assume all the parameters to be real. The CP-violating case (see [4] for a review) is interesting in its own right as it can significantly modify the phenomenology (see for example ref. [17] and references therein). We will leave the CP-violating case for a future study.
} 
four models described in table 1. Our analysis shows that only models of Type X ("lepton specific") survive all these constraints.

The paper is organised as follows. In section 2 we present a detailed analysis of the EW constraints on the masses of the pseudoscalar boson $A$, charged scalar $H^{ \pm}$, and additional neutral heavy scalar $H$. We study radiative corrections in the 2HDMs and, in particular, the impact of the precise measurements of the $W$ boson mass $M_{W}$ and the effective weak mixing angle $\sin ^{2} \theta_{\text {eff }}^{\text {lept }}$. It is then important to check whether a large mass hierarchy between $A$ and $H^{ \pm}$is allowed by the Higgs measurements at the LHC and by the theoretical constraints on vacuum stability and perturbativity, which is discussed in section 3. In section 4 we present the additional contributions of the 2 HDMs to the muon $g-2$ and discuss their implications on the four types of model analysed in this paper. Prospects for constraints from the electron $g-2$ are presented in section 5. Conclusions are drawn in section 6 .

\section{Electroweak constraints}

In this section we analyze the constraints arising from EW precision observables on 2HDMs. In particular, we compare the theoretical $2 \mathrm{HDM}$ s predictions for $M_{W}$ and $\sin ^{2} \theta_{\mathrm{eff}}^{\text {lept }}$ with their present experimental values via a combined $\chi^{2}$ analysis [35].

As it was shown for the first time in [36], in the SM the $W$ mass can be computed perturbatively by means of the following relation

$$
M_{W}^{2}=\frac{M_{Z}^{2}}{2}\left[1+\sqrt{1-\frac{4 \pi \alpha_{\mathrm{em}}}{\sqrt{2} G_{F} M_{Z}^{2}} \frac{1}{1-\Delta r}}\right],
$$

where $\alpha_{\mathrm{em}}$ is the fine-structure constant, $G_{F}$ is the Fermi constant and $M_{Z}$ is the $Z$ boson mass. The on-shell quantity $\Delta r$ [36], representing the radiative corrections, is a function of the parameters of the SM. In particular, since $\Delta r$ also depends on $M_{W}$, eq. (2.1) can be solved in an iterative way. The relation between the effective weak mixing angle $\sin ^{2} \theta_{\mathrm{eff}}^{\text {lept }}$ and the on-shell weak mixing angle $\sin ^{2} \theta_{W}$ is given by [37]

$$
\sin ^{2} \theta_{\mathrm{eff}}^{\text {lept }}=k_{l}\left(M_{Z}^{2}\right) \sin ^{2} \theta_{W},
$$

where $\sin ^{2} \theta_{W}=1-M_{W}^{2} / M_{Z}^{2}[36]$ and $k_{l}\left(q^{2}\right)=1+\Delta k_{l}\left(q^{2}\right)$ is the real part of the vertex form factor $Z \rightarrow l \bar{l}$ evaluated at $q^{2}=M_{Z}^{2}$.

The $2 \mathrm{HDM} \mathcal{O}\left(\alpha_{\mathrm{em}}\right)$ corrections to $\Delta r$ and $\Delta k_{l}$ can be written in form

$$
\begin{aligned}
& \Delta r^{2 \mathrm{HDM}}=\Delta r+\Delta \tilde{r}, \\
& \Delta k_{l}^{2 \mathrm{HDM}}=\Delta k_{l}+\Delta \tilde{k}_{l},
\end{aligned}
$$

where the tilded quantities indicate the additional 2HDM contributions not contained in the SM prediction. These additional corrections depend only on the particles and parameters of the extended Higgs sector which are not present in the SM part. The radiative corrections $\Delta r$ and $\Delta k_{l}$ are known up to two-loop order, including some partial higher-order EW and QCD corrections [38, 39] (for a review of these corrections we refer the reader to [40]). For 
our purposes, this level of accuracy in the SM part is not needed, and in our codes [35] we implemented the full one-loop SM result plus the leading two-loop contributions of [4143]. The additional correction $\Delta \tilde{r}$ has been available for a long time [44]. We recalculated this contribution and found agreement with the previous results. The additional 2HDM correction $\Delta \tilde{k}_{l}$ was not available in the literature. We evaluated it following the notation of [44]. For convenience, the calculation was carried out in the $\overline{\mathrm{MS}}$ scheme and then translated to the on-shell scheme by means of the relations derived in $[37,45]$. The analytic results can be found in [35]. Following the analysis of [44], we neglected the $\mathcal{O}(\alpha)$ corrections where a virtual Higgs is attached to an external fermion line, since they are suppressed by factors of $\mathcal{O}\left(M_{f} / M_{W}\right)$. As a result, no new contributions to vertex and box diagrams are present with respect to the SM ones. All the additional diagrams fall in the class of bosonic self-energies and $\gamma-Z$ mixing terms. We point out that, in this approximation, these EW constraints do not depend on the way fermions couple to the Higgs bosons and, therefore, all four types of $2 \mathrm{HDM}$ discussed in this paper share the same EW constraints.

The $2 \mathrm{HDM}$ predictions for $M_{W}$ and $\sin ^{2} \theta_{\text {eff }}^{\text {lept }}$ depend on the $Z$ boson mass $M_{Z}=$ $91.1876(21) \mathrm{GeV}$ [16], the top quark mass, $m_{t}=173.2(0.9) \mathrm{GeV}$ [46], the strong coupling constant $\alpha_{s}\left(M_{Z}\right)=0.1185$ (6) [16], the variation of the fine-structure constant due to light quarks, $\Delta \alpha_{\text {had }}^{(5)}\left(M_{Z}^{2}\right)=0.02763$ (14) [47], the masses of the neutral Higgs bosons $M_{h}=126 \mathrm{GeV}, M_{H}$ and $M_{A}$, the charged Higgs mass $M_{H^{ \pm}}$, and the combination $(\beta-\alpha)$ of the mixing angles in the scalar sector, which we will set to $\pi / 2$ to be consistent with the LHC results on Higgs boson searches [18-34]. To analyze the constraints on 2HDMs arising from the present measurements of $M_{W}$ and $\sin ^{2} \theta_{\text {eff }}^{\text {lept }}$ we define

$$
\chi_{\mathrm{EW}}^{2}=\left(\frac{M_{W}^{2 \mathrm{HDM}}-M_{W}^{\mathrm{EXP}}}{\sigma_{M_{W}}^{\mathrm{EXP}}}\right)^{2}+\left(\frac{\sin ^{2} \theta_{\mathrm{eff}}^{\mathrm{lept}, 2 \mathrm{HDM}}-\sin ^{2} \theta_{\mathrm{eff}}^{\mathrm{lept}, \mathrm{EXP}}}{\sigma_{\sin ^{2} \theta_{W}}^{\mathrm{EXP}}}\right)^{2},
$$

and use the following experimental values for $M_{W}[16]$ and $\sin ^{2} \theta_{\text {eff }}^{\text {lept }}[48]$ :

$$
\begin{aligned}
M_{W}^{\mathrm{EXP}} & =80.385 \pm 0.015 \mathrm{GeV}, \\
\sin ^{2} \theta_{\text {eff }}^{\text {lept,EXP }} & =0.23153 \pm 0.00016 .
\end{aligned}
$$

We note that the corrections $\Delta r^{2 \mathrm{HDM}}$ and $\Delta k_{l}^{2 \mathrm{HDM}}$ implemented in our code receive a large contribution from the well-known quantity $\Delta \rho^{2 \mathrm{HDM}}=\Delta \rho+\Delta \tilde{\rho}$ :

$$
\begin{aligned}
& \Delta r^{2 \mathrm{HDM}}=\Delta \alpha^{2 \mathrm{HDM}}-\frac{\cos ^{2} \theta_{W}}{\sin ^{2} \theta_{W}} \Delta \rho^{2 \mathrm{HDM}}+\ldots, \\
& \Delta k_{l}^{2 \mathrm{HDM}}=+\frac{\cos ^{2} \theta_{W}}{\sin ^{2} \theta_{W}} \Delta \rho^{2 \mathrm{HDM}}+\ldots,
\end{aligned}
$$

where $\Delta \alpha^{2 \mathrm{HDM}}$ is the photon vacuum polarization contribution in the $2 \mathrm{HDM}$. The definition of the parameter $\Delta \rho$, consistent with eqs. (2.7), (2.8), can be found in [49].

The results of our analysis are displayed in figure 1, where we chose three different values of the charged scalar mass, $M_{H^{ \pm}}=200,400$ and $600 \mathrm{GeV}$, the Higgs-to-gauge boson coupling $\beta-\alpha=\pi / 2, M_{h}=126 \mathrm{GeV}$, and we set $M_{Z}, m_{t}, \alpha_{s}\left(M_{Z}\right)$ and $\Delta \alpha_{\text {had }}^{(5)}\left(M_{Z}^{2}\right)$ to their experimental central values. The green, yellow and gray regions of the plane $M_{A}$ vs. 

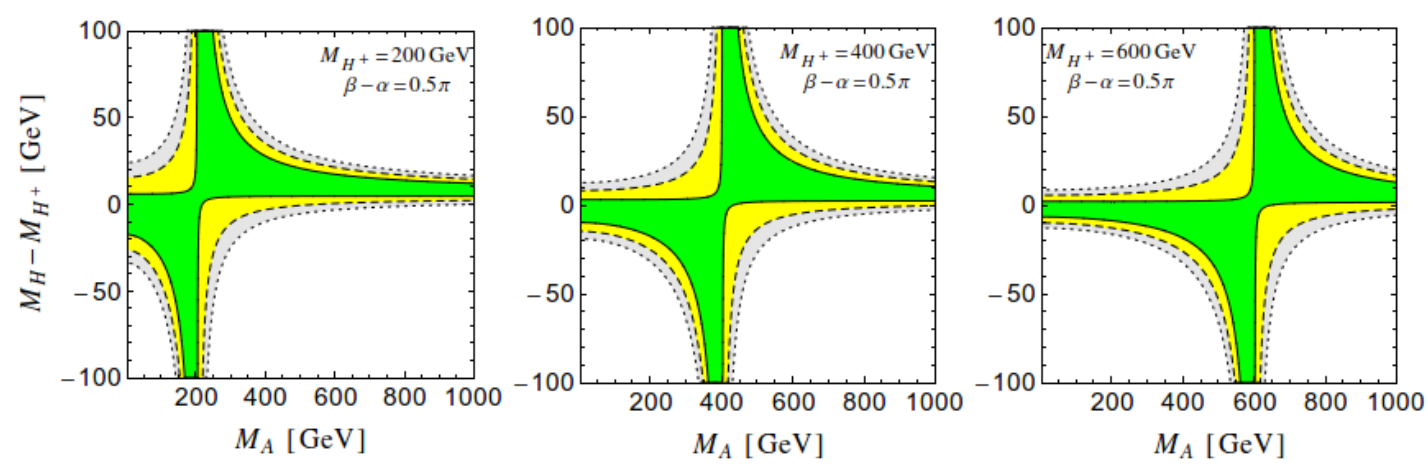

Figure 1. The parameter space allowed in the $M_{A}$ vs. $\Delta M_{H}=M_{H}-M_{H^{ \pm}}$plane by EW precision constraints $\left(M_{W}\right.$ and $\left.\sin ^{2} \theta_{\text {eff }}^{\text {lept }}\right)$. The green, yellow, gray regions satisfy $\Delta \chi_{\mathrm{EW}}^{2}\left(M_{A}, \Delta M\right)<$ $2.3,6.2,11.8$, corresponding to $68.3,95.4$, and $99.7 \%$ confidence intervals, respectively. From left to right, different values of $M_{H^{ \pm}}=200,400$ and $600 \mathrm{GeV}$ are shown. All plots employ $\beta-\alpha=\pi / 2$ and $M_{h}=126 \mathrm{GeV}$, and $M_{Z}, m_{t}, \alpha_{s}\left(M_{Z}\right)$ and $\Delta \alpha_{\text {had }}^{(5)}\left(M_{Z}^{2}\right)$ are set to their measured central values.

$\Delta M_{H}=M_{H}-M_{H^{ \pm}}$where drawn requiring $\Delta \chi_{\mathrm{EW}}^{2}\left(M_{A}, \Delta M\right)=\chi_{\mathrm{EW}}^{2}\left(M_{A}, \Delta M\right)-\chi_{\mathrm{EW}, \min }^{2}<$ $2.3,6.2,11.8$, respectively, which are the critical values corresponding to the $68.3,95.4$, and $99.7 \%$ confidence intervals $\left(\chi_{\mathrm{EW}, \mathrm{min}}^{2}\right.$ is the absolute minimum of $\left.\chi_{\mathrm{EW}}^{2}\left(M_{A}, \Delta M\right)\right)[16,50]$. Note that in the case of a large splitting between $M_{H}$ and $M_{H^{ \pm}}, M_{A}$ is required to be almost degenerate with $M_{H^{ \pm}}$in order to satisfy the EW constraints. This point has already been remarked upon in [23, 29] (see also [51] for alternative conditions to satisfy the EW constraints). In addition, we observe that all values of $M_{A}$ are allowed when $M_{H}$ and $M_{H^{ \pm}}$are almost degenerate. This useful result will be used in section 4 .

\section{Theoretical constraints on the splitting $M_{A^{-}} M_{H^{+}}$}

Although, as shown in the previous section, any value of $M_{A}$ is allowed by the EW precision tests in the limit of $M_{H} \sim M_{H^{ \pm}}$, a large separation between $M_{H^{ \pm}}$and $M_{A}$ is strongly constrained by theoretical considerations of vacuum stability and perturbativity. Since we are interested in a light pseudoscalar (motivated by the resolution of the muon $g-2$ discrepancy), it is important to check how small $M_{A}$ is allowed to be. In this section we study such constraints in a semi-analytical way.

The CP-conserving $2 \mathrm{HDM}$ with softly broken $Z_{2}$ symmetry is parametrized by seven real parameters, namely $\lambda_{1, \ldots, 5}, m_{12}^{2}$ and $\tan \beta[4,5]$. The general scalar potential of two Higgs doublets $\Phi_{1}$ and $\Phi_{2}$ is given by

$$
\begin{aligned}
V= & m_{11}^{2}\left|\Phi_{1}\right|^{2}+m_{22}^{2}\left|\Phi_{2}\right|^{2}-m_{12}^{2}\left(\Phi_{1}^{\dagger} \Phi_{2}+\Phi_{2}^{\dagger} \Phi_{1}\right) \\
& +\frac{\lambda_{1}}{2}\left|\Phi_{1}\right|^{4}+\frac{\lambda_{2}}{2}\left|\Phi_{2}\right|^{4}+\lambda_{3}\left|\Phi_{1}\right|^{2}\left|\Phi_{2}\right|^{2}+\lambda_{4}\left|\Phi_{1}^{\dagger} \Phi_{2}\right|^{2}+\frac{\lambda_{5}}{2}\left[\left(\Phi_{1}^{\dagger} \Phi_{2}\right)^{2}+\left(\Phi_{2}^{\dagger} \Phi_{1}\right)^{2}\right],
\end{aligned}
$$

where the Higgs vacuum expectation values are given by $\left\langle\Phi_{1,2}\right\rangle=\frac{1}{\sqrt{2}}\left(0, v_{1,2}\right)^{T}$. The masses of all the physical Higgs bosons and the mixing angle $\alpha$ between CP-even neutral ones are obtained from $\tan \beta$ and the remaining six real parameters [5]. The vacuum stability and perturbativity conditions put bounds on these parameters and correlate the masses 
of different neutral and charged scalars. For example, the vacuum stability condition requires [5]:

$$
\lambda_{1,2}>0, \quad \lambda_{3}>-\sqrt{\lambda_{1} \lambda_{2}}, \quad\left|\lambda_{5}\right|<\lambda_{3}+\lambda_{4}+\sqrt{\lambda_{1} \lambda_{2}},
$$

and the requirement of global minimum is imposed by the condition [52]

$$
m_{12}^{2}\left(m_{11}^{2}-m_{22}^{2} \sqrt{\lambda_{1} / \lambda_{2}}\right)\left(\tan \beta-\left(\lambda_{1} / \lambda_{2}\right)^{1 / 4}\right)>0,
$$

where $m_{11}$ and $m_{22}$ are functions of $\lambda_{i}, m_{12}$ and $\tan \beta$ as expressed in ref. [5]. For the perturbativity criterion, we will consider three different values for the maximum couplings

$$
\left|\lambda_{i}\right| \lesssim\left|\lambda_{\max }\right|=\sqrt{4 \pi}, 2 \pi, 4 \pi,
$$

to see their impact on the allowed mass spectrum. A large separation between any two scalar masses in $2 \mathrm{HDM}$ is controlled by the above constraints.

For a given value of $\tan \beta$, one can express two of the six parameters, namely $\lambda_{4}$ and $\lambda_{5}$, entirely in terms of physical masses $M_{A}, M_{H^{ \pm}}$and the parameter $m_{12}$ using the relations [5]

$$
\begin{aligned}
M_{A}^{2} & =\frac{m_{12}^{2}}{\sin \beta \cos \beta}-\lambda_{5} v^{2}, \\
M_{H^{ \pm}}^{2} & =M_{A}^{2}+\frac{1}{2} v^{2}\left(\lambda_{5}-\lambda_{4}\right) .
\end{aligned}
$$

Furthermore, for a given value of $\tan \beta$ and solving for the $M_{h}, M_{H}$ and the SM-like Higgs coupling limit $\beta-\alpha=\pi / 2$, one can obtain semi-analytical solutions for the remaining four real parameters in terms of four physical masses and the only free parameter $\lambda_{1}$ using the expressions given in ref. [5]. The expressions for $\lambda_{2,3}$ valid for $\tan \beta \gg 1$ are

$$
\begin{aligned}
\lambda_{2} v^{2} & \simeq M_{h}^{2}+\lambda_{1} v^{2} / \tan ^{4} \beta, \\
\lambda_{3} v^{2} & \simeq 2 M_{H^{ \pm}}^{2}-2 M_{H}^{2}+M_{h}^{2}+\lambda_{1} v^{2} / \tan ^{2} \beta, \\
m_{12}^{2} & \simeq M_{H}^{2} / \tan \beta+\left(M_{h}^{2}-\lambda_{1} v^{2}\right) / \tan ^{3} \beta .
\end{aligned}
$$

We find that in the $\beta-\alpha=\pi / 2$ limit the parameters $\lambda_{2,3}$ depend negligibly on $\tan \beta$. Similar expressions for $\lambda_{4,5}$ can be obtained using eq. (3.5). One can now impose the conditions (3.2), (3.3) and (3.4) on the above equations. As can be seen from eq. (3.5), the difference $M_{H^{ \pm}}^{2}-M_{A}^{2}$ is proportional to $\lambda_{5}-\lambda_{4}$ and it is restricted to be smaller than $\lambda_{3}+\sqrt{\lambda_{1} \lambda_{2}}$ as required by vacuum stability condition, eq. (3.2). Both $\lambda_{2}$ and $\lambda_{3}$ have almost negligible dependence on $\lambda_{1}$ as can be seen from the semi-analytic expressions in eqs. (3.6). Taking $M_{h}=126 \mathrm{GeV}, \lambda_{1}=\lambda_{\max }$ and imposing all the theoretical constraints mentioned above, one gets the regions allowed in $M_{A^{-}} M_{ \pm}$plane as shown in figure 2.

The plots in figure 2 depend very mildly on $\tan \beta$ so that similar results hold for any value of $\tan \beta \in[5,100]$. We also note that the change in the allowed regions is negligible with respect to small departures from the SM-like Higgs coupling limit $\beta-\alpha=\pi / 2$. One can clearly see that for a light pseudoscalar with $M_{A} \lesssim 100 \mathrm{GeV}$ the charged Higgs boson mass gets an upper bound of $M_{H^{ \pm}} \lesssim 200 \mathrm{GeV}$. Also, figure 2 shows the presence of lower bounds on $M_{A}$ if the charged Higgs boson mass is heavier than $\sim 200 \mathrm{GeV}$. We will discuss the implications of these correlations in the following sections. 

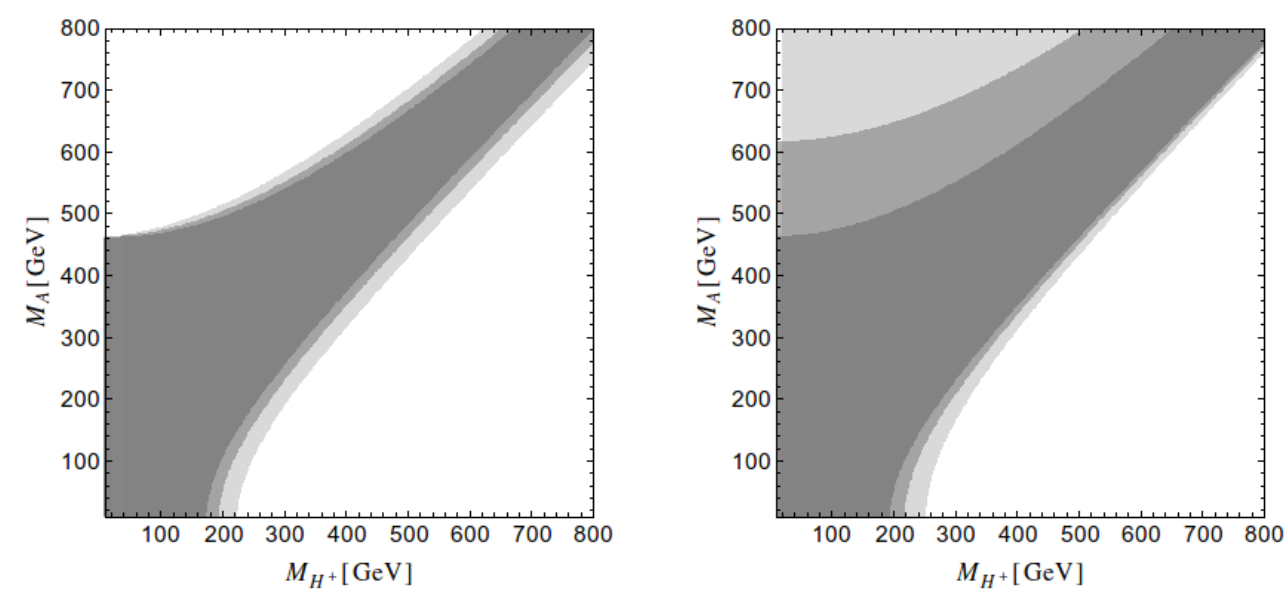

Figure 2. Theoretical constraints on the $M_{A^{-}} M_{H^{ \pm}}$plane in $2 \mathrm{HDMs}$ with softly broken $Z_{2}$ symmetry. We employ $\beta-\alpha=\pi / 2$ and $M_{h}=126 \mathrm{GeV}$. The darker to lighter gray regions in the left panel correspond to the allowed regions for $\Delta M \equiv M_{H}-M_{H^{ \pm}}=\{20,0,-30\} \mathrm{GeV}$ and $\lambda_{\max }=\sqrt{4 \pi}$. The allowed regions in the right panel correspond to $\lambda_{\max }=\{\sqrt{4 \pi}, 2 \pi, 4 \pi\}$ and vanishing $\Delta M$. Both plots are obtained for $\tan \beta=50$, but the change with respect to values of $\tan \beta \in[5,100]$ is negligible.

\section{Constraints from the muon $g-2$}

The SM prediction of the muon $g-2$ is conveniently split into QED, EW and hadronic contributions: $a_{\mu}^{\mathrm{SM}}=a_{\mu}^{\mathrm{QED}}+a_{\mu}^{\mathrm{EW}}+a_{\mu}^{\mathrm{H}}$. The QED prediction, computed up to five loops, currently stands at $a_{\mu}^{\mathrm{QED}}=116584718.951(80) \times 10^{-11}$ [53], while the EW effects provide $a_{\mu}^{\mathrm{EW}}=153.6(1.0) \times 10^{-11}[54-56]$. The latest calculations of the hadronic leading order contribution, via the hadronic $e^{+} e^{-}$annihilation data, are in agreement: $a_{\mu}^{\mathrm{HLO}}=6903(53) \times$ $10^{-11}$ [57], $6923(42) \times 10^{-11}$ [58] and $6949(43) \times 10^{-11}$ [47]. The next-to-leading order hadronic term is further divided into two parts: $a_{\mu}^{\mathrm{HNLO}}=a_{\mu}^{\mathrm{HNLO}}(\mathrm{vp})+a_{\mu}^{\mathrm{HNLO}}(\mathrm{lbl})$. The first one, $-98.4(7) \times 10^{-11}$ [47], is the $O\left(\alpha^{3}\right)$ contribution of diagrams containing hadronic vacuum polarization insertions [59]. The second term, also of $O\left(\alpha^{3}\right)$, is the leading hadronic light-by-light contribution; the latest calculations of this term, $105(26) \times 10^{-11}[60]$ and $116(39) \times 10^{-11}$ [57], are in good agreement, and an intense research program is under way to improve its evaluation [61-64]. Very recently, also the next-to-next-to leading order hadronic corrections have been determined: insertions of hadronic vacuum polarizations were computed to be $a_{\mu}^{\mathrm{HNNLO}}(\mathrm{vp})=12.4(1) \times 10^{-11}$ [65], while hadronic light-by-light corrections have been estimated to be $a_{\mu}^{\mathrm{HNNLO}}(\mathrm{lbl})=3(2) \times 10^{-11}$ [66]. If we add the value $a_{\mu}^{\mathrm{HLO}}=6903(53) \times 10^{-11}$ of [58] (which roughly coincides with the average of the three hadronic leading order values reported above) to the conservative estimate $a_{\mu}^{\mathrm{HNLO}}(\mathrm{lbl})=$ $116(39) \times 10^{-11}$ of [57] and the rest of the other SM contributions, we obtain

$$
a_{\mu}^{\mathrm{SM}}=116591829(57) \times 10^{-11}
$$

(for reviews of $a_{\mu}^{\mathrm{SM}}$ see $[57,67-71]$ ). The difference between $a_{\mu}^{\mathrm{SM}}$ and the experimental value $[72]$

$$
a_{\mu}^{\mathrm{EXP}}=116592091(63) \times 10^{-11}
$$


is, therefore, $\Delta a_{\mu} \equiv a_{\mu}^{\mathrm{EXP}}-a_{\mu}^{\mathrm{SM}}=+262(85) \times 10^{-11}$, i.e. $3.1 \sigma$ (all errors were added in quadrature).

Models with two Higgs doublets give additional contributions to $a_{\mu}$ which could bridge the above discrepancy $\Delta a_{\mu}[11-15]$. All the Higgs bosons of the 2HDMs contribute to $a_{\mu}$. However, in order to explain $\Delta a_{\mu}$, their total contribution should be positive and, as we will see, enhanced by $\tan \beta$. In the $2 \mathrm{HDM}$, the one-loop contributions to $a_{\mu}$ of the neutral and charged Higgs bosons are [73-75]

$$
\delta a_{\mu}^{2 \text { HDM }}(1 \text { loop })=\frac{G_{F} m_{\mu}^{2}}{4 \pi^{2} \sqrt{2}} \sum_{j}\left(y_{\mu}^{j}\right)^{2} r_{\mu}^{j} f_{j}\left(r_{\mu}^{j}\right),
$$

where $j=\left\{h, H, A, H^{ \pm}\right\}, r_{\mu}^{j}=m_{\mu}^{2} / M_{j}^{2}$, and

$$
\begin{aligned}
f_{h, H}(r) & =\int_{0}^{1} d x \frac{x^{2}(2-x)}{1-x+r x^{2}}, \\
f_{A}(r) & =\int_{0}^{1} d x \frac{-x^{3}}{1-x+r x^{2}}, \\
f_{H^{ \pm}}(r) & =\int_{0}^{1} d x \frac{-x(1-x)}{1-(1-x) r} .
\end{aligned}
$$

The normalized Yukawa couplings $y_{\mu}^{h, H, A}$ are listed in table 1 , and $y_{\mu}^{H^{ \pm}}=y_{\mu}^{A}$. The one-loop contribution of the light CP-even boson $h$ is given by eq. (4.3) with $j=h$; however, as we work in the limit $\beta-\alpha \approx \pi / 2$ in which $h$ has the same couplings as the SM Higgs boson, its contribution is already contained in $a_{\mu}^{\mathrm{EW}}$ and shouldn't therefore be included in the additional $2 \mathrm{HDM}$ contribution (in any case, this contribution is negligible: setting $M_{h}=126 \mathrm{GeV}$ and $y_{\mu}^{h}=1$ we obtain $\left.2 \times 10^{-14}\right)$. The formulae in eqs. (4.3)-(4.6) show that the one-loop contributions to $a_{\mu}$ are positive for the neutral scalars $h$ and $H$, and negative for the pseudo-scalar and charged Higgs bosons $A$ and $H^{ \pm}$(for $M_{H^{ \pm}}>m_{\mu}$ ). In the limit $r \ll 1$,

$$
\begin{aligned}
f_{h, H}(r) & =-\ln r-7 / 6+O(r), \\
f_{A}(r) & =+\ln r+11 / 6+O(r), \\
f_{H^{ \pm}}(r) & =-1 / 6+O(r),
\end{aligned}
$$

showing that in this limit $f_{H^{ \pm}}(r)$ is suppressed with respect to $f_{h, H, A}(r)$.

The one-loop results in eqs. (4.7)-(4.9) also show that, in the limit $r \ll 1, \delta a_{\mu}^{2 \mathrm{HDM}}$ (1loop) roughly scales with the fourth power of the muon mass. For this reason, two-loop effects may become relevant if one can avoid the suppression induced by these large powers of the muon mass. This is indeed the case for two-loop Barr-Zee type diagrams with effective $h \gamma \gamma$, $H \gamma \gamma$ or $A \gamma \gamma$ vertices generated by the exchange of heavy fermions [11]. Their contribution to the muon $g-2$ is $[11,12,15,54]$

$$
\delta a_{\mu}^{2 \mathrm{HDM}}(2 \mathrm{loop}-\mathrm{BZ})=\frac{G_{F} m_{\mu}^{2}}{4 \pi^{2} \sqrt{2}} \frac{\alpha_{\mathrm{em}}}{\pi} \sum_{i, f} N_{f}^{c} Q_{f}^{2} y_{\mu}^{i} y_{f}^{i} r_{f}^{i} g_{i}\left(r_{f}^{i}\right),
$$


where $i=\{h, H, A\}, r_{f}^{i}=m_{f}^{2} / M_{i}^{2}$, and $m_{f}, Q_{f}$ and $N_{f}^{c}$ are the mass, electric charge and number of color degrees of freedom of the fermion $f$ in the loop. The functions $g_{i}(r)$ are

$$
g_{i}(r)=\int_{0}^{1} d x \frac{\mathcal{N}_{i}(x)}{x(1-x)-r} \ln \frac{x(1-x)}{r},
$$

where $\mathcal{N}_{h, H}(x)=2 x(1-x)-1$ and $\mathcal{N}_{A}(x)=1$. As in the one-loop case, the two-loop Barr-Zee contribution of the light scalar $h$ is given by the formula in eq. (4.10) with $i=h$ but, once again, working in the limit $\beta-\alpha \approx \pi / 2$, its contribution is already contained in $a_{\mu}^{\mathrm{EW}}$ and we will therefore not include it in the additional 2HDM contribution (setting $M_{h}=126 \mathrm{GeV}, y_{\mu}^{h}=y_{f}^{h}=1$ and summing over top, bottom and tau lepton loops we obtain $\left.-1.4 \times 10^{-11}\right)$. Note the enhancement factor $m_{f}^{2} / m_{\mu}^{2}$ of the two-loop formula in eq. (4.10) relative to the one-loop contribution in eq. (4.3). As this factor $m_{f}^{2} / m_{\mu}^{2}$ can overcome the additional loop suppression factor $\alpha / \pi$, the two-loop contributions in eq. (4.10) may become larger than the one-loop ones. Moreover, the signs of the two-loop functions $g_{h, H}$ (negative) and $g_{A}$ (positive) for the CP-even and CP-odd contributions are opposite to those of the functions $f_{h, H}$ (positive) and $f_{A}$ (negative) at one-loop. In type II models in the limit $\beta-\alpha=\pi / 2$, a numerical calculation shows that for a light scalar with mass lower than $\sim 5 \mathrm{GeV}$ and $\tan \beta \gtrsim 5$ the negative two-loop scalar contribution is smaller in magnitude than the positive one-loop result; also, for $M_{A} \gtrsim 3 \mathrm{GeV}$ the positive two-loop pseudoscalar contribution is larger than the negative one-loop result. A light pseudoscalar with $M_{A} \gtrsim 3 \mathrm{GeV}$ can therefore generate a sizeable positive contribution which can account for the observed $\Delta a_{\mu}$ discrepancy. ${ }^{2}$ A similar conclusion is valid for the pseudoscalar contribution in type $\mathrm{X}$ models [78]. In fact, we notice from the pseudoscalar Yukawa couplings in table 1 that the contribution of the tau lepton loop is enhanced by a factor $\tan ^{2} \beta$ both in type II and in X models; on the contrary, it is suppressed by $1 / \tan ^{2} \beta$ in models of type I and Y.

The additional $2 \mathrm{HDM}$ contribution $\delta a_{\mu}^{2 \mathrm{HDM}}=\delta a_{\mu}^{2 \mathrm{HDM}}(1 \mathrm{loop})+\delta a_{\mu}^{2 \mathrm{HDM}}(2 \mathrm{loop}-\mathrm{BZ})$ obtained adding eqs. (4.3) and (4.10) (without the $h$ contributions) is compared with $\Delta a_{\mu}$ in figure 3 for type II and X models as a function of $\tan \beta$ and $M_{A}$. Once again, we used the SM coupling limit $\beta-\alpha=\pi / 2$. In both models, relatively small $M_{A}$ values are needed to generate the positive pseudoscalar contribution to $a_{\mu}$ required to bridge the $\Delta a_{\mu}$ discrepancy. In turn, in order to satisfy the theoretical constraints of section 3 for a light pseudoscalar with $M_{A} \lesssim 100 \mathrm{GeV}$, the charged Higgs mass must be lower than $\sim 200 \mathrm{GeV}$, as shown in figure 2, but anyway larger that the model-independent LEP bound $M_{H^{ \pm}} \gtrsim 79 \mathrm{GeV}$ [16]. Under these conditions, the EW constraints discussed in section 2 restrict the value of the neutral scalar mass to be $M_{H} \sim M_{H^{ \pm}}$(see figure 1). We therefore chose the conservative values $M_{H}=M_{H^{ \pm}}=200 \mathrm{GeV}$ to draw figure 3. Slightly higher values of $\tan \beta$ would be preferred in figure 3 if the lower values $M_{H}=M_{H^{ \pm}}=150 \mathrm{GeV}$ were chosen instead (in fact, a lower $M_{H}$ induces a slightly larger negative scalar contribution to $a_{\mu}$ ). For given values of $M_{A}$ and $\tan \beta$, the contribution to $\delta a_{\mu}^{2 \mathrm{HDM}}$ in type II models is slightly higher than that in type $\mathrm{X}$ models because of the additional $\tan ^{2} \beta$ enhancement

\footnotetext{
${ }^{2}$ One could also advocate a very light scalar with mass lower than $\sim 5 \mathrm{GeV}$, but this scenario is challenged experimentally [76] (see also [77]).
} 

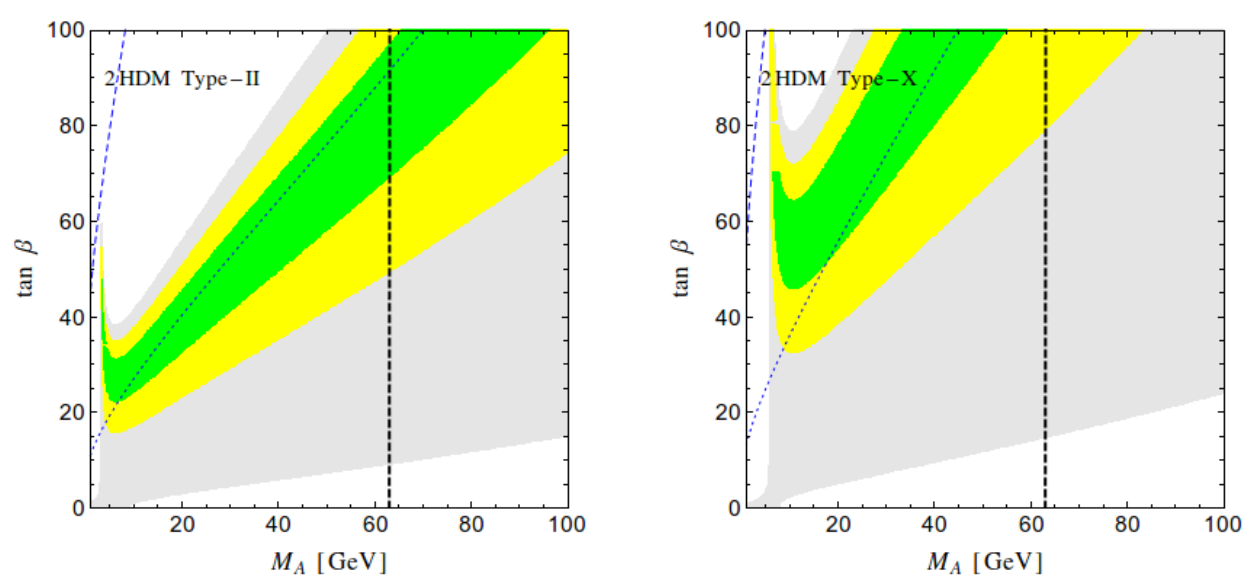

Figure 3. The $1 \sigma, 2 \sigma$ and $3 \sigma$ regions allowed by $\Delta a_{\mu}$ in the $M_{A^{-}}$tan $\beta$ plane taking the limit of $\beta-\alpha=\pi / 2$ and $M_{h(H)}=126(200) \mathrm{GeV}$ in type II (left panel) and type X (right panel) 2HDMs. The regions below the dashed (dotted) lines are allowed at $3 \sigma(1.4 \sigma)$ by $\Delta a_{e}$. The vertical dashed line corresponds to $M_{A}=M_{h} / 2$ (see text for an explanation).

for the down-type quark contribution. It is important to note that, on the contrary, type I and Y models cannot account for the present value of $\Delta a_{\mu}$ due to their lack of $\tan ^{2} \beta$ enhancements.

In 2HDMs of type II (and Y) a very stringent limit can be set on $M_{H^{ \pm}}$from the flavour observables $\operatorname{Br}\left(\bar{B} \rightarrow X_{s} \gamma\right)$ and $\Delta m_{B_{s}}$, as well as from the hadronic $Z \rightarrow b \bar{b}$ branching ratio $R_{b}: M_{H^{ \pm}}>380 \mathrm{GeV}$ at $95 \% \mathrm{CL}$ irrespective of the value of $\tan \beta$ [79-82]. This bound is much stronger than the model-independent one obtained at LEP, $M_{H^{ \pm}}>79.3 \mathrm{GeV}$ at 95\% CL $[16,83]$. This strong constraint $M_{H^{ \pm}}>380 \mathrm{GeV}$, combined with the theoretical requirements shown in figure 2 , leads to $M_{A} \gtrsim 300 \mathrm{GeV}$. In turn, this lower bound on $M_{A}$ is in conflict with the required value for $\Delta a_{\mu}$, as can be seen from figure 3. Therefore, type II models are strongly disfavoured by these combined constraints. On the other hand, no such strong flavour bounds on $M_{H^{ \pm}}$exist in type $\mathrm{X}$ models [4, 81]. These models are therefore consistent with all the constraints we considered, provided $M_{A}$ is small and $\tan \beta$ large (see figure 3), $M_{H^{ \pm}} \lesssim 200 \mathrm{GeV}$ (figure 2), and $M_{H} \sim M_{H^{ \pm}}$(figure 1).

Finally, a sufficiently light pseudoscalar may lead to the decay $h \rightarrow A A$ with potentially large branching fraction even in the SM decoupling limit [78]. It will be thus interesting to perform a dedicated analysis for such a process by considering the further decays of light $A$ in the type-X $2 \mathrm{HDM}$. We do not perform such analysis here and leave it for future studies. However, we would like to emphasize that any limits arising from this process can still be avoided by considering the region $M_{A}>M_{h} / 2$. From figure 3 , we see that even if $M_{A}>M_{h} / 2$, there is still sufficient parameter space left which can provide an explanation to the excess in the $(g-2)_{\mu}$.

\section{Constraints from the electron $g-2$}

It is usually believed that new-physics contributions to the electron $g-2\left(a_{e}\right)$ are too small to be relevant; with this assumption, the measurement of $a_{e}$ is equated with the SM 
prediction to determine the value of the fine-structure constant $\alpha_{\mathrm{em}}: a_{e}^{\mathrm{SM}}\left(\alpha_{\mathrm{em}}\right)=a_{e}^{\mathrm{EXP}}$. However, as discussed in [84], in the last few years the situation has been changing thanks to several theoretical [85] and experimental [86] advancements in the determination of $a_{e}$ and, at the same time, to new independent measurements of $\alpha_{\mathrm{em}}$ obtained from atomic physics experiments [87]. The error induced in the theoretical prediction $a_{e}^{\mathrm{SM}}\left(\alpha_{\mathrm{em}}\right)$ by the experimental uncertainty of $\alpha_{\mathrm{em}}$ (used as an input, rather than an output), although still dominating, has been significantly reduced, and one can start to view $a_{e}$ as a probe of physics beyond the SM.

The present difference between the SM prediction and the experimental value is $\Delta a_{e} \equiv$ $a_{e}^{\mathrm{EXP}}-a_{e}^{\mathrm{SM}}=-10.5(8.1) \times 10^{-13}$, i.e. 1.3 standard deviations, thus providing a beautiful test of QED. We note that the sign of $\Delta a_{e}$ is opposite to that of $\Delta a_{\mu}$ (although the uncertainty is still large). The uncertainty $8.1 \times 10^{-13}$ is dominated by that of the SM prediction through the error caused by the uncertainty of $\alpha_{\mathrm{em}}$, but work is in progress to reduce it significantly [88]. Following the analysis presented above for the muon $g-2$, we compared $\Delta a_{e}$ with the $2 \mathrm{HDM}$ contribution $\delta a_{e}^{2 \mathrm{HDM}}=\delta a_{e}^{2 \mathrm{HDM}}(1 \mathrm{loop})+\delta a_{e}^{2 \mathrm{HDM}}(2$ loop $-\mathrm{BZ})$ obtained adding eqs. (4.3) and (4.10), obviously replacing $m_{\mu}$ and $y_{\mu}^{j}$ with $m_{e}$ and $y_{e}^{j}$. The result is shown once again in figure 3 , for type II and X models, as a function of $\tan \beta$ and $M_{A}$. In each panel, the region below the dashed (dotted) line is the $3 \sigma(1.4 \sigma)$ region allowed by $\Delta a_{e}$. Clearly, the precision of $\Delta a_{e}$ is not yet sufficient to play a significant role in limiting the 2HDMs, but this will change with new, more precise, measurements of $a_{e}$ and $\alpha_{\mathrm{em}}$. For example, reducing the uncertainty of $\Delta a_{e}$ by a factor of three and maintaining its present (negative) central value, the $3 \sigma$ regions allowed by $\Delta a_{e}$ completely disappear from both panels of figure 3. In fact, at present, increasing by $1 \sigma$ the negative central value $\Delta a_{e}=-10.5 \times 10^{-13}$, one still gets a negative value, which cannot be accounted for in the region of parameter space shown in figure 3. (Increasing the present central value by $1.4 \sigma$ one gets $+0.8 \times 10^{-13}$, which is the input used to draw the dotted lines in figure 3.) Obviously, future tests will depend both on the uncertainty and on the central value of $\Delta a_{e}$.

\section{Conclusions}

In recent times there has been renewed interest in the phenomenology of models with two Higgs doublets. Most of the focus has been on four possible variations of them, namely, type I, II, X (or "lepton specific") and Y ("flipped"). In this work we presented a detailed phenomenological analysis with the aim of challenging these four models. We included constraints from electroweak precision tests, vacuum stability and perturbativity, direct searches at colliders, muon and electron $g-2$, and constraints from $B$-physics observables. In these models, all the Higgses couple similarly to the gauge bosons, but differently to the fermions. Therefore, the electroweak constraints (along with the perturbativity and vacuum stability ones) are common to all of them, while the rest of the constraints vary from model to model. Using a stringent set of precision electroweak measurements we showed that, in the limit $(\beta-\alpha) \rightarrow \pi / 2$ consistent with the LHC results on Higgs boson searches, all values of $M_{A}$ are allowed when $M_{H}$ and $M_{H^{ \pm}}$are almost degenerate. We 
considered a CP-conserving scenario where the $126 \mathrm{GeV}$ resonance discovered at the $\mathrm{LHC}$ has been identified with the lightest CP-even boson $h$.

The 2HDM predictions for observables which depend on fermion couplings are expected to vary from model to model. In fact, the interplay between the muon $g-2$ and $b \rightarrow s \gamma$ is the key distinguisher between the various types. A light pseudoscalar with couplings proportional to $\tan \beta$ is required to explain the discrepancy between the SM prediction and the observed value of the muon $g-2$. This is only possible in type II and X models. On the other hand, in type II and Y models the $\mathrm{BR}(b \rightarrow s \gamma)$ sets a strong $\mathcal{O}(380 \mathrm{GeV})$ lower bound on the mass of the charged scalar which, taken together with the perturbativity and vacuum stability constraints, was shown to leave hardly any space for a light pseudoscalar. On the contrary, only loose constraints arise from the $\mathrm{BR}(b \rightarrow s \gamma)$ in type I and X models, because both up and down type quarks couple to the same Higgs doublet in these models. Therefore, we showed that type X models are the only ones which can accommodate the muon $g-2$ without violating the $\operatorname{BR}(b \rightarrow s \gamma)$ and the rest of the present constraints. We also noted that an improved measurement of the electron $g-2$ may lead in the future to further significant bounds on $2 \mathrm{HDM}$.

The parameter space favourable for the muon $g-2$ in type $\mathrm{X}$ models is quite limited in mass ranges for the heavy neutral and charged scalar: $M_{H} \sim M_{H^{ \pm}} \lesssim 200 \mathrm{GeV}$ (with small $M_{A}$ and large $\tan \beta$ ). These bosons can be searched for in forthcoming collider experiments, even if this parameter region could be elusive because the productions of the additional Higgs bosons $A, H$, and $H^{ \pm}$are suppressed either by $1 / \tan ^{2} \beta$ (in single productions, e.g. through gluon fusion) or by $\cos (\beta-\alpha)$ (associated productions of $V \phi$ and $h \phi$ ). The leading search channels for the extra bosons would then be pair or associated productions through $p p \rightarrow \gamma / Z / W \rightarrow H^{+} H^{-} / H A / H^{ \pm} A / H^{ \pm} H$ followed by the decays $H^{ \pm} \rightarrow l^{ \pm} \nu$ and $A, H \rightarrow l^{+} l^{-}$, which can be readily tested at the next run of the LHC $[4,89]$.

\section{Acknowledgments}

We would like to thank G. Degrassi, T. Dorigo, A. Ferroglia, P. Paradisi, A. Sirlin and G. Venanzoni for very useful discussions. MP and KMP also thank the Department of Physics and Astronomy of the University of Padova for its support. Their work was supported in part by the PRIN 2010-11 of the Italian MIUR and by the European Program INVISIBLES (PITN-GA-2011-289442). MP and SKV thank the hospitality of KIAS, Seoul, where this work started. SKV also thanks DST, Govt. of India, for the support through the project SR/S2/RJN-25/2008.

Open Access. This article is distributed under the terms of the Creative Commons Attribution License (CC-BY 4.0), which permits any use, distribution and reproduction in any medium, provided the original author(s) and source are credited.

\section{References}

[1] ATLAS collaboration, Observation of a new particle in the search for the standard model Higgs boson with the ATLAS detector at the LHC, Phys. Lett. B 716 (2012) 1 [arXiv: 1207.7214] [INSPIRE]. 
[2] CMS collaboration, Observation of a new boson at a mass of $125 \mathrm{GeV}$ with the CMS experiment at the LHC, Phys. Lett. B 716 (2012) 30 [arXiv:1207.7235] [INSPIRE].

[3] J.F. Gunion, H.E. Haber, G.L. Kane and S. Dawson, The Higgs hunter's guide, Front. Phys. 80 (2000) 1 [INSPIRE].

[4] G.C. Branco et al., Theory and phenomenology of two-Higgs-doublet models, Phys. Rept. 516 (2012) 1 [arXiv: 1106.0034] [INSPIRE].

[5] J.F. Gunion and H.E. Haber, The CP conserving two Higgs doublet model: the approach to the decoupling limit, Phys. Rev. D 67 (2003) 075019 [hep-ph/0207010] [INSPIRE].

[6] M. Aoki, S. Kanemura, K. Tsumura and K. Yagyu, Models of Yukawa interaction in the two Higgs doublet model and their collider phenomenology, Phys. Rev. D 80 (2009) 015017 [arXiv: 0902.4665] [INSPIRE].

[7] A. Pich and P. Tuzon, Yukawa alignment in the two-Higgs-doublet model, Phys. Rev. D 80 (2009) 091702 [arXiv:0908.1554] [INSPIRE].

[8] E. Ma, Verifiable radiative seesaw mechanism of neutrino mass and dark matter, Phys. Rev. D 73 (2006) 077301 [hep-ph/0601225] [INSPIRE].

[9] R. Barbieri, L.J. Hall and V.S. Rychkov, Improved naturalness with a heavy Higgs: an alternative road to LHC physics, Phys. Rev. D 74 (2006) 015007 [hep-ph/0603188] [INSPIRE].

[10] A. Crivellin, A. Kokulu and C. Greub, Flavor-phenomenology of two-Higgs-doublet models with generic Yukawa structure, Phys. Rev. D 87 (2013) 094031 [arXiv:1303.5877] [INSPIRE].

[11] D. Chang, W.-F. Chang, C.-H. Chou and W.-Y. Keung, Large two loop contributions to g-2 from a generic pseudoscalar boson, Phys. Rev. D 63 (2001) 091301 [hep-ph/0009292] [INSPIRE].

[12] K.-m. Cheung, C.-H. Chou and O.C.W. Kong, Muon anomalous magnetic moment, two Higgs doublet model and supersymmetry, Phys. Rev. D 64 (2001) 111301 [hep-ph/0103183] [INSPIRE].

[13] M. Krawczyk, Precision muon $g-2$ results and light Higgs bosons in the 2HDM(II), Acta Phys. Polon. B 33 (2002) 2621 [hep-ph/0208076] [INSPIRE].

[14] F. Larios, G. Tavares-Velasco and C.P. Yuan, A very light CP odd scalar in the two Higgs doublet model, Phys. Rev. D 64 (2001) 055004 [hep-ph/0103292] [INSPIRE].

[15] K. Cheung and O.C.W. Kong, Can the two Higgs doublet model survive the constraint from the muon anomalous magnetic moment as suggested?, Phys. Rev. D 68 (2003) 053003 [hep-ph/0302111] [INSPIRE].

[16] Particle Data Group collaboration, K. Olive et al., Review of particle physics, Chin. Phys. C 38 (2014) 090001.

[17] L. Basso et al., Probing the charged Higgs boson at the LHC in the CP-violating type-II 2HDM, JHEP 11 (2012) 011 [arXiv: 1205.6569] [INSPIRE].

[18] W. Altmannshofer, S. Gori and G.D. Kribs, A minimal flavor violating 2HDM at the LHC, Phys. Rev. D 86 (2012) 115009 [arXiv:1210.2465] [InSPIRE].

[19] S. Chang et al., Comprehensive study of two Higgs doublet model in light of the new boson with mass around $125 \mathrm{GeV}$, JHEP 05 (2013) 075 [arXiv:1210.3439] [INSPIRE]. 
[20] C.-Y. Chen and S. Dawson, Exploring two Higgs doublet models through Higgs production, Phys. Rev. D 87 (2013) 055016 [arXiv: 1301.0309] [inSPIRE].

[21] C.-Y. Chen, S. Dawson and M. Sher, Heavy Higgs searches and constraints on two Higgs doublet models, Phys. Rev. D 88 (2013) 015018 [arXiv:1305.1624] [INSPIRE].

[22] D. López-Val, T. Plehn and M. Rauch, Measuring extended Higgs sectors as a consistent free couplings model, JHEP 10 (2013) 134 [arXiv:1308.1979] [INSPIRE].

[23] O. Eberhardt, U. Nierste and M. Wiebusch, Status of the two-Higgs-doublet model of type-II, JHEP 07 (2013) 118 [arXiv: 1305.1649] [INSPIRE].

[24] A. Barroso, 2HDM at the LHC - The story so far, arXiv:1304.5225 [INSPIRE].

[25] G. Bélanger, B. Dumont, U. Ellwanger, J.F. Gunion and S. Kraml, Global fit to Higgs signal strengths and couplings and implications for extended Higgs sectors, Phys. Rev. D 88 (2013) 075008 [arXiv: 1306 . 2941] [INSPIRE].

[26] V. Barger, L.L. Everett, H.E. Logan and G. Shaughnessy, Scrutinizing the $125 \mathrm{GeV}$ Higgs boson in two Higgs doublet models at the LHC, ILC and Muon Collider, Phys. Rev. D 88 (2013) 115003 [arXiv:1308.0052] [INSPIRE].

[27] S. Chang et al., Two Higgs doublet models for the LHC Higgs boson data at $\sqrt{s}=7$ and $8 \mathrm{TeV}$, JHEP 09 (2014) 101 [arXiv:1310.3374] [INSPIRE].

[28] K. Cheung, J.S. Lee and P.-Y. Tseng, Higgcision in the two-Higgs doublet models, JHEP 01 (2014) 085 [arXiv: 1310.3937] [INSPIRE].

[29] A. Celis, V. Ilisie and A. Pich, Towards a general analysis of LHC data within two-Higgs-doublet models, JHEP 12 (2013) 095 [arXiv:1310.7941] [INSPIRE].

[30] J. Baglio, O. Eberhardt, U. Nierste and M. Wiebusch, Benchmarks for Higgs pair production and heavy Higgs searches in the two-Higgs-doublet model of type II, Phys. Rev. D 90 (2014) 015008 [arXiv: 1403.1264 ] [INSPIRE].

[31] X.-D. Cheng, Y.-D. Yang and X.-B. Yuan, Phenomenological discriminations of the Yukawa interactions in two-Higgs doublet models with $Z_{2}$ symmetry, Eur. Phys. J. C 74 (2014) 3081 [arXiv: 1401.6657] [INSPIRE].

[32] P.M. Ferreira et al., The CP-conserving 2HDM after the 8 TeV run, arXiv:1407.4396 [INSPIRE].

[33] B. Dumont, J.F. Gunion, Y. Jiang and S. Kraml, Constraints on and future prospects for two-Higgs-doublet models in light of the LHC Higgs signal, Phys. Rev. D 90 (2014) 035021 [arXiv: 1405.3584] [INSPIRE].

[34] P.S.B. Dev and A. Pilaftsis, Maximally symmetric two Higgs doublet model with natural standard model alignment, arXiv:1408.3405 [INSPIRE].

[35] A. Broggio, Quantum effects in the two Higgs doublet model, M.Sc. thesis, University of Padua, Italy (2009).

[36] A. Sirlin, Radiative corrections in the $\mathrm{SU}(2)_{L} \times \mathrm{U}(1)$ theory: a simple renormalization framework, Phys. Rev. D 22 (1980) 971 [InSPIRE].

[37] P. Gambino and A. Sirlin, Relation between $\sin ^{2} \theta_{W}\left(M_{Z}\right)$ and $\sin ^{2} \theta_{\mathrm{eff}}^{\text {lept }}$, Phys. Rev. D 49 (1994) 1160 [hep-ph/9309326] [INSPIRE].

[38] M. Awramik, M. Czakon, A. Freitas and G. Weiglein, Precise prediction for the $W$ boson mass in the standard model, Phys. Rev. D 69 (2004) 053006 [hep-ph/0311148] [INSPIRE]. 
[39] M. Awramik, M. Czakon and A. Freitas, Electroweak two-loop corrections to the effective weak mixing angle, JHEP 11 (2006) 048 [hep-ph/0608099] [INSPIRE].

[40] A. Sirlin and A. Ferroglia, Radiative corrections in precision electroweak physics: a historical perspective, Rev. Mod. Phys. 85 (2013) 263 [arXiv:1210.5296] [INSPIRE].

[41] G. Degrassi, P. Gambino and A. Sirlin, Precise calculation of $M_{W}, \sin ^{2} \theta_{W}\left(M_{Z}\right)$ and $\sin ^{2} \theta_{\text {eff }}^{\text {(lept }}$, Phys. Lett. B 394 (1997) 188 [hep-ph/9611363] [INSPIRE].

[42] G. Degrassi, P. Gambino, M. Passera and A. Sirlin, The role of $M_{W}$ in precision studies of the standard model, Phys. Lett. B 418 (1998) 209 [hep-ph/9708311] [INSPIRE].

[43] A. Ferroglia, G. Ossola, M. Passera and A. Sirlin, Simple formulae for $\sin ^{2} \theta_{\mathrm{eff}}^{\text {lept }}, M_{W}, \Gamma_{l}$ and their physical applications, Phys. Rev. D 65 (2002) 113002 [hep-ph/0203224] [INSPIRE].

[44] S. Bertolini, Quantum effects in a two Higgs doublet model of the electroweak interactions, Nucl. Phys. B 272 (1986) 77 [INSPIRE].

[45] G. Degrassi, S. Fanchiotti and A. Sirlin, Relations between the on-shell and Ms frameworks and the $M_{W}-M-Z$ interdependence, Nucl. Phys. B 351 (1991) 49 [INSPIRE].

[46] CDF Collaboration, D0 collaboration, T. Aaltonen et al., Combination of the top-quark mass measurements from the Tevatron collider, Phys. Rev. D 86 (2012) 092003 [arXiv: 1207.1069] [INSPIRE].

[47] K. Hagiwara, R. Liao, A.D. Martin, D. Nomura and T. Teubner, $(g-2)_{\mu}$ and $\alpha\left(M_{Z}^{2}\right)$ re-evaluated using new precise data, J. Phys. G 38 (2011) 085003 [arXiv:1105.3149] [INSPIRE].

[48] ALEPH, DELPHi, L3, OPAL, SLD, LEP Electroweak Working Group, SLD Electroweak Group, SLD Heavy Flavour Group collaboration, S. Schael et al., Precision electroweak measurements on the $Z$ resonance, Phys. Rept. 427 (2006) 257 [hep-ex/0509008] [INSPIRE].

[49] F. Jegerlehner, Physics of precision experiments with Zs, Prog. Part. Nucl. Phys. 27 (1991) 1 [INSPIRE].

[50] W.A. Rolke, A.M. Lopez and J. Conrad, Limits and confidence intervals in the presence of nuisance parameters, Nucl. Instrum. Meth. A 551 (2005) 493 [physics/0403059] [INSPIRE].

[51] J.-M. Gerard and M. Herquet, A twisted custodial symmetry in the two-Higgs-doublet model, Phys. Rev. Lett. 98 (2007) 251802 [hep-ph/0703051] [INSPIRE].

[52] A. Barroso, P.M. Ferreira, I.P. Ivanov and R. Santos, Metastability bounds on the two Higgs doublet model, JHEP 06 (2013) 045 [arXiv: 1303.5098] [INSPIRE].

[53] T. Aoyama, M. Hayakawa, T. Kinoshita and M. Nio, Complete tenth-order QED contribution to the muon g-2, Phys. Rev. Lett. 109 (2012) 111808 [arXiv:1205.5370] [INSPIRE].

[54] A. Czarnecki, B. Krause and W.J. Marciano, Electroweak fermion loop contributions to the muon anomalous magnetic moment, Phys. Rev. D 52 (1995) 2619 [hep-ph/9506256] [INSPIRE].

[55] A. Czarnecki, B. Krause and W.J. Marciano, Electroweak corrections to the muon anomalous magnetic moment, Phys. Rev. Lett. 76 (1996) 3267 [hep-ph/9512369] [INSPIRE].

[56] C. Gnendiger, D. Stöckinger and H. Stöckinger-Kim, The electroweak contributions to $(g-2)_{\mu}$ after the Higgs boson mass measurement, Phys. Rev. D 88 (2013) 053005 [arXiv: 1306.5546] [INSPIRE]. 
[57] F. Jegerlehner and A. Nyffeler, The muon g-2, Phys. Rept. 477 (2009) 1 [arXiv:0902.3360] [INSPIRE].

[58] M. Davier, A. Hoecker, B. Malaescu and Z. Zhang, Reevaluation of the hadronic contributions to the muon $g-2$ and to $\alpha\left(M_{Z}\right)$, Eur. Phys. J. C 71 (2011) 1515 [Erratum ibid. C 72 (2012) 1874] [arXiv: 1010.4180] [INSPIRE].

[59] B. Krause, Higher order hadronic contributions to the anomalous magnetic moment of leptons, Phys. Lett. B 390 (1997) 392 [hep-ph/9607259] [INSPIRE].

[60] J. Prades, E. de Rafael and A. Vainshtein, Hadronic light-by-light scattering contribution to the muon anomalous magnetic moment, arXiv:0901.0306 [INSPIRE].

[61] G. Colangelo, M. Hoferichter, M. Procura and P. Stoffer, Dispersive approach to hadronic light-by-light scattering, JHEP 09 (2014) 091 [arXiv: 1402.7081] [INSPIRE].

[62] G. Colangelo, M. Hoferichter, B. Kubis, M. Procura and P. Stoffer, Towards a data-driven analysis of hadronic light-by-light scattering, Phys. Lett. B 738 (2014) 6 [arXiv:1408.2517] [INSPIRE].

[63] T. Blum, S. Chowdhury, M. Hayakawa and T. Izubuchi, Hadronic light-by-light scattering contribution to the muon anomalous magnetic moment from lattice QCD, arXiv:1407.2923 [INSPIRE].

[64] V. Pauk and M. Vanderhaeghen, Anomalous magnetic moment of the muon in a dispersive approach, arXiv: 1409.0819 [INSPIRE].

[65] A. Kurz, T. Liu, P. Marquard and M. Steinhauser, Hadronic contribution to the muon anomalous magnetic moment to next-to-next-to-leading order, arXiv:1403.6400 [INSPIRE].

[66] G. Colangelo, M. Hoferichter, A. Nyffeler, M. Passera and P. Stoffer, Remarks on higher-order hadronic corrections to the muon g-2, Phys. Lett. B 735 (2014) 90 [arXiv: 1403.7512] [INSPIRE].

[67] T. Blum et al., The muon $(g-2)$ theory value: present and future, arXiv:1311.2198 [INSPIRE].

[68] K. Melnikov and A. Vainshtein, Theory of the muon anomalous magnetic moment, Springer Tracts in Modern Physics volume 216, Springer, Germany (2006).

[69] M. Davier and W.J. Marciano, The theoretical prediction for the muon anomalous magnetic moment, Ann. Rev. Nucl. Part. Sci. 54 (2004) 115 [inSPIRE].

[70] M. Passera, The standard model prediction of the muon anomalous magnetic moment, J. Phys. G 31 (2005) R75 [hep-ph/0411168] [INSPIRE].

[71] M. Knecht, The anomalous magnetic moment of the muon: a theoretical introduction, Lect. Notes Phys. 629 (2004) 37 [hep-ph/0307239] [INSPIRE].

[72] Muon G-2 collaboration, G.W. Bennett et al., Final report of the muon E821 anomalous magnetic moment measurement at BNL, Phys. Rev. D 73 (2006) 072003 [hep-ex/0602035] [INSPIRE].

[73] B. Lautrup, A. Peterman and E. de Rafael, Recent developments in the comparison between theory and experiments in quantum electrodynamics, Phys. Rept. 3 (1972) 193 [INSPIRE].

[74] J.P. Leveille, The second order weak correction to $(g-2)$ of the muon in arbitrary gauge models, Nucl. Phys. B 137 (1978) 63 [InSPIRE]. 
[75] A. Dedes and H.E. Haber, Can the Higgs sector contribute significantly to the muon anomalous magnetic moment?, JHEP 05 (2001) 006 [hep-ph/0102297] [INSPIRE].

[76] P. Franzini et al., Limits on Higgs bosons, scalar quarkonia and $\eta(B)$ 's from radiative $\Upsilon$ decays, Phys. Rev. D 35 (1987) 2883 [InSPIRE].

[77] K. Schmidt-Hoberg, F. Staub and M.W. Winkler, Constraints on light mediators: confronting dark matter searches with B physics, Phys. Lett. B 727 (2013) 506 [arXiv: 1310.6752] [INSPIRE].

[78] J. Cao, P. Wan, L. Wu and J.M. Yang, Lepton-specific two-Higgs doublet model: experimental constraints and implication on Higgs phenomenology, Phys. Rev. D 80 (2009) 071701 [arXiv: 0909.5148] [INSPIRE].

[79] M. Misiak et al., Estimate of $B\left(\bar{B} \rightarrow X_{s} \gamma\right)$ at $O\left(\alpha_{s}^{2}\right)$, Phys. Rev. Lett. 98 (2007) 022002 [hep-ph/0609232] [INSPIRE].

[80] O. Deschamps et al., The two Higgs doublet of type II facing flavour physics data, Phys. Rev. D 82 (2010) 073012 [arXiv:0907.5135] [INSPIRE].

[81] F. Mahmoudi and O. Stal, Flavor constraints on the two-Higgs-doublet model with general Yukawa couplings, Phys. Rev. D 81 (2010) 035016 [arXiv:0907.1791] [InSPIRE].

[82] T. Hermann, M. Misiak and M. Steinhauser, $\bar{B} \rightarrow X_{s} \gamma$ in the two Higgs doublet model up to next-to-next-to-leading order in QCD, JHEP 11 (2012) 036 [arXiv:1208.2788] [INSPIRE].

[83] ALEPH collaboration, A. Heister et al., Search for charged Higgs bosons in $e^{+} e^{-}$collisions at energies up to $\sqrt{s}=209 \mathrm{GeV}$, Phys. Lett. B 543 (2002) 1 [hep-ex/0207054] [INSPIRE].

[84] G.F. Giudice, P. Paradisi and M. Passera, Testing new physics with the electron g- 2, JHEP 11 (2012) 113 [arXiv:1208.6583] [INSPIRE].

[85] T. Aoyama, M. Hayakawa, T. Kinoshita and M. Nio, Tenth-order QED contribution to the electron $g-2$ and an improved value of the fine structure constant, Phys. Rev. Lett. 109 (2012) 111807 [arXiv:1205.5368] [INSPIRE].

[86] D. Hanneke, S. Fogwell and G. Gabrielse, New measurement of the electron magnetic moment and the fine structure constant, Phys. Rev. Lett. 100 (2008) 120801 [arXiv:0801.1134] [INSPIRE].

[87] R. Bouchendira, P. Clade, S. Guellati-Khelifa, F. Nez and F. Biraben, New determination of the fine structure constant and test of the quantum electrodynamics, Phys. Rev. Lett. 106 (2011) 080801 [arXiv: 1012.3627] [INSPIRE].

[88] F. Terranova and G.M. Tino, Testing the $a_{\mu}$ anomaly in the electron sector through a precise measurement of $h / M$, Phys. Rev. A 89 (2014) 052118 [arXiv:1312.2346] [INSPIRE].

[89] S. Kanemura, K. Tsumura, K. Yagyu and H. Yokoya, Fingerprinting non-minimal Higgs sectors, arXiv: 1406.3294 [INSPIRE]. 